\title{
A Broadband Conducted Emission Behavior Modeling Method of Active Devices Based on Vector Fitting Theory
}

\author{
Xi Chen, Yao Chen \\ EMC lab \\ School of Electronic and Information Engineering, Beihang University \\ Beijing, China \\ chenxi@buaa.edu.cn
}

\begin{abstract}
This paper presents a methodology about building the active device broadband behavior model that can prediction of its conducted emission accurately. The architecture of the model is composed of two sub-models: the Passive Distribution Network (PDN) sub-model and the Internal Source (IS) sub-model. An example shows that the model of the device can be useful into the general duty simulator, while also preserving the accuracy of the model.
\end{abstract}

Keywords-actice device; broadband behavior model; vector fitting (VF) technology; conducted emission; 'black box' model

\section{INTRODUCTION}

With modern electronic components are increasingly complex, the electromagnetic compatibility of the devices is becoming increasingly important. A few years ago the focus of attention is often focused on the PCB traces, crystal and interconnects. But in recent years, researchers found that the circuit board de-vice will produce electromagnetic interference and also the victim of electromagnetic interference. And now the focus of study from the previous system-level electromagnetic compatibility, slowly turned to lower device chip level.

There are two ways to describe the active de-vices: one is based on the components of the works, get the physical device models and parameters are closely related to the device works. The SPICE model is the most widely used model. The advantage of the first way is that it has a high accuracy, and with the development of modeling means and the technology of semiconductor, people have been able to provide this model in a variety levels to meet different accura-cy needs. The disadvantage of the model is that the model is complex, long computing time and modeling cycle, besides that this modeling method is not universal, only for device manufacturers, for the avera-ge user is not feasible. Another method is from the el-ectrical characteristics of the components, the components is seen as a 'black box', then measuring the electrical characteristics of its ports, extract the device model, without involving the device works, the method is called behavioral modeling. This model is representative of IBIS models[1] and Sparameters models. The Advantage of the method is versatile, short time waste, a wide suitable range, in high-frequency nonlinear or high-power case behavioral models is virtually the only option. The disadvantage is that the accuracy is poor.

This paper proposes a method of modeling method using the data which measuring from the scattering parameters of the DUT active device then build the device conducted emission behavioral mo-del. Among this to modeling device passive distribution network (PDN) is using vector fitting method by measurement though Vector Network Analyzer (VNA) then through measure or estimate method to establish the device's internal source.

\section{THE ACTIVE DEVICE CONDUCTED EMISSION BEHAVIOR MODEL}

According the structural characteristics of the active device, the model has two sub-models:

\section{A. Passive distribution network (PDN) sub-model}

The sub-model description the terminal impedan-ce between the power supply terminal and I/O ports, including the internal power traces: on-chip metal line, the internal decoupling capacitors between power and ground plane and the device package (wire and interconnect). PDN model is also used to describe the $\mathrm{I} / \mathrm{O}$ power supply, as well as how they are connected to other parts of the chip and PCB.

Vector fitting (VF) technology was first proposed by Norwegian scholars B. Gustavsen, used for power transmission system transient modeling and simulat-ion of electromagnetic interference, the vector fitting technique is widely used in the passive system modeling 
and time-domain simulation power integrity and EMC simulation field. Here the vector fitting technique in order to establish accurate active device passive distribution network model used in the PDN modeling of the active device.

\section{1) Principles of vector fitting}

Vector fitting is one of a rational approximation technique, use the port frequency response data for the minimum mean square error (LMS) fitting then get the following rational polynomial.

$$
H_{\text {Mea }}(s) \cong H_{F i t}(s)=\sum_{i=1}^{N} \frac{\hat{r}_{i}}{s-p_{l}}+\hat{d}+s \hat{e}
$$

$p_{1}, \hat{r_{i}}, \hat{d}, e$ is the data that we need fitting, $p_{l}$ is the plural form of single real numbers or conjugate, the process of Vector fitting is as follows:

Set an initial set of poles $P_{0}=\left(\bar{p}_{1}, \bar{p}_{2} \ldots \ldots . \bar{p}_{N}\right)$ and $P_{0}$ covering all bands and then set the iteration function:

$$
\begin{gathered}
\sigma(s)=1+\sum_{i=1}^{N} \frac{\tilde{r}_{i}}{s-\bar{p}_{i}} \\
\sigma(s) H_{F i t}(s)=\sum_{i=1}^{N} \frac{\hat{r}_{i}}{s-\bar{p}_{l}}+\hat{d}+s \hat{e}
\end{gathered}
$$

The first two equations required have the same poles $\bar{p}_{l}$, so, the zeros of the $\sigma(s)$ is also the poles of the $H_{F i t}(s)$. Put the (2) into (3) and then get the

$$
\left(1+\sum_{i=1}^{N} \frac{\tilde{r}_{i}}{s-\bar{p}_{i}}\right) H_{F i t}(s) \approx \sum_{i=1}^{N} \frac{\hat{r}_{i}}{s-\bar{p}_{l}}+\hat{d}+s \hat{e}
$$

Then bring $H_{\text {Mea }}\left(s_{k}\right), k=1,2 \ldots \ldots . . m$ into equation (4) to replace $H_{F i t}\left(s_{k}\right), k=1,2 \ldots \ldots . m$ and get a overdetermined linear equation about $\hat{r}_{i}^{\wedge}, \hat{d}, e$. For the sampling frequency $s_{k}$ we can obtain the following linear equations: $A X=b$ (5), among this:

$$
\begin{aligned}
& A=\left[\begin{array}{cccccccc}
\frac{1}{s_{1}-\bar{p}_{1}} & \cdots & \frac{1}{s_{1}-\bar{p}_{N}} & 1 & s_{1} & \frac{-H_{\text {Mea }}\left(s_{1}\right)}{s_{1}-\bar{p}_{1}} & \cdots & \frac{-H_{\text {Mee }}\left(s_{1}\right)}{s_{1}-\bar{p}_{N}} \\
\cdots & \cdots & \cdots & \cdots & \cdots & \cdots & \cdots & \cdots \\
\frac{1}{s_{m}-\bar{p}_{1}} & \cdots & \frac{1}{s_{m}-\bar{p}_{N}} & 1 & s_{m} & \frac{-H_{\text {Mea }}\left(s_{1}\right)}{s_{m}-\bar{p}_{1}} & \cdots & \frac{-H_{\text {Mea }}\left(s_{1}\right)}{s_{m}-\bar{p}_{N}}
\end{array}\right]_{(k \times 2(N+1))} \\
& X=\left(\hat{r}_{1}, \hat{r}_{2} \cdots r_{N}, \hat{d}, e, \tilde{r}_{1}, \tilde{r}_{2} \cdots \tilde{r}_{N}\right)^{T} \subset C^{2 N+2} \\
& b=\left(H_{\text {Mea }}\left(s_{1}\right), H_{\text {Mea }}\left(s_{2}\right) \cdots H_{\text {Mea }}\left(s_{m}\right)\right)^{T} \subset C^{m}
\end{aligned}
$$

Through LMSE or matrix QR decomposition method can obtain the unknown variables $X$. After obtain the parameters $\hat{r}_{i}^{\hat{y}}, \hat{d}, e, \tilde{r}_{i}$, the equation (3) can write as pole-zeros: $\frac{\prod_{i=1}^{N+1}\left(s-\hat{z}_{i}\right)}{\prod_{i=1}^{N+1}\left(s-\bar{p}_{i}\right)} \hat{e}=H_{F i t}(s) \frac{\prod_{i=1}^{N+1}\left(s-\tilde{z}_{i}\right)}{\prod_{i=1}^{N+1}\left(s-\bar{p}_{i}\right)}$,so
$H_{F i t}(s)=\hat{e} \frac{\prod_{i=1}^{N+1}\left(s-\hat{z}_{i}\right)}{\prod_{i=1}^{N}\left(s-\tilde{z}_{i}\right)}(9)$. Then another set of poles can be obtained: $P_{t}=\left(\tilde{z}_{1}, \tilde{z}_{2} \cdots \tilde{z}_{N}\right)$. And $P_{t}$ as the initial poles into the formula and repeat (5)-(9) until convergence $\left(P_{t} \rightarrow P_{\infty}=\left(p_{1}, p_{2} \cdots p_{N}\right)\right)$. Then we will get the stability of the pole vector $P_{\infty}$ into the formula $H_{\text {Fit }}(s)$ can be obtained $\hat{r}_{i}, \hat{d}, e$.

\section{2) Passivity}

Because the PDN net must satisfy the passivity requirement, the behavioral model by vector fitting must meet the passive requirements. The admittance $\overline{\bar{Y}}(s)$ parameter gets from vector fitting theory need to satisfy the passivity necessary and sufficient condition for[3]:

a) $\overline{\bar{Y}}(s)$ in the right half of the complex plane ( $r e\{s\}>0$ ) should be defined and analytical;

b) $\overline{\bar{H}}(s)$ is conjugate symmetric;

c) $\overline{\bar{Y}}^{H}(s)+\overline{\bar{Y}} \geq 0$

For VF expression is analytical, and the coefficients of the numerator and denominator of the expression are both real coefficients, so that a), b) are already satisfied, the c) is the key to meet the passive requirement.

\section{3) Passivity determine}

There we use the time domain passivity deter-mine methods, this method only calculate a matrix eigenvalue once and then it can find all the illegal pa-ssive frequency area, defined decision matrix[4]:

$$
\overline{\bar{P}}=\overline{\bar{A}}\left(\overline{\overline{B D}} \overline{\bar{D}}^{-1} \overline{\bar{C}}-\overline{\bar{A}}\right)
$$

Among this $\overline{\bar{A}}, \overline{\bar{B}}, \overline{\bar{C}}, \overline{\bar{D}}$ is the matrix coefficients of time domain state equation and the determination method is: If system judgment matrix $\overline{\bar{P}}$ exist positive real eigenvalues $\omega^{2}$, so the frequency $\omega$ is a cross point between passive and active, otherwise the model to meet the passive.

\section{4) Passivity enhancement}

If the behavioral model built in violation of passivity, then it need to be passivity enhancement it can make the residue of the eigenvalue around the pole around the model eventually meet the passive.

\section{5) Create the time-domain circuit netlist}

S-parameter models and the residue pole model can only use in a single simulation with the simulator, it cannot be separated from the simulation environment, therefore, in order to make the model can be applied in more universal circuit emulator, here will be created the behavioral model transformed into a time domain circuit SPICE netlist. 

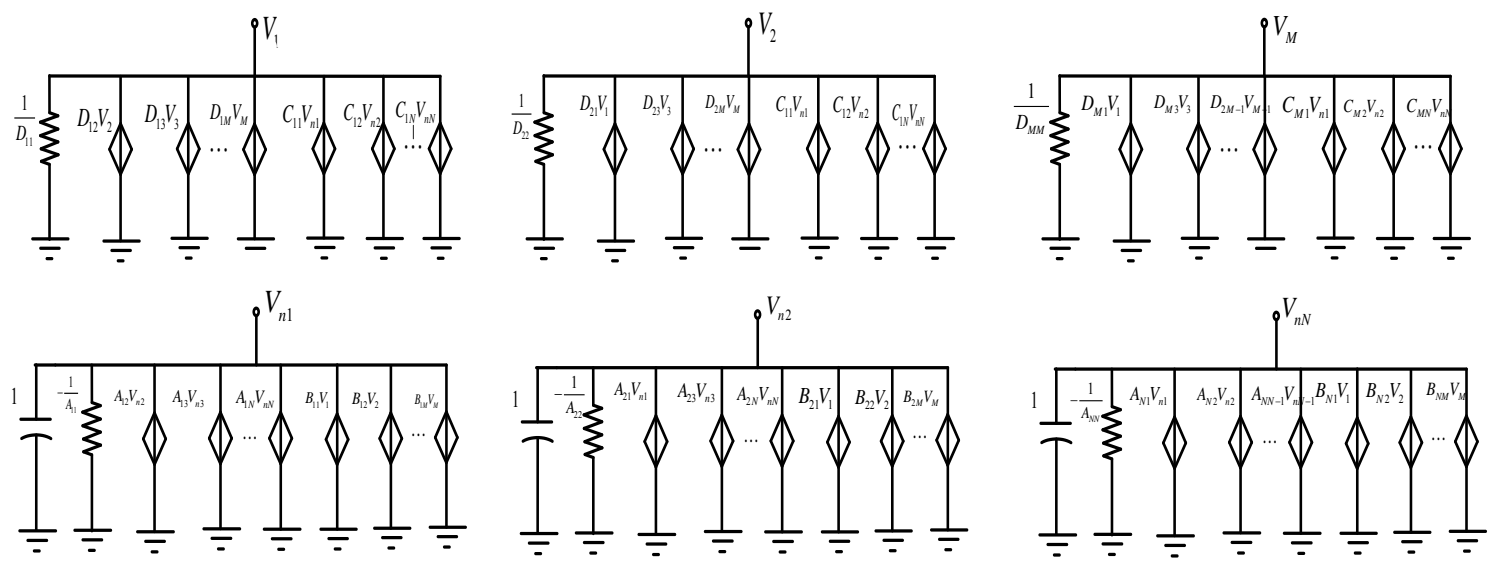

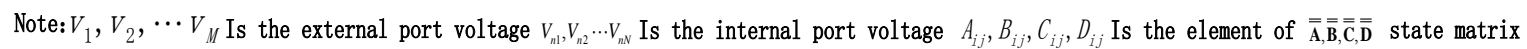
$\diamond$ Is the voltage controlled current source.

Fig. 1 SPICE netlist building use the fitting result of state equation parameters

Creating a SPICE netlist, may use the time-domain equation of state as follows[6]:

$$
\left\{\begin{array}{c}
\frac{d \mathbf{x}(t)}{d t}=\overline{\bar{A}} \mathbf{x}(t)+\overline{\bar{B}} \mathbf{u}(t) \\
\mathbf{y}(t)=\overline{\bar{C}} \mathbf{x}(t)+\overline{\bar{D}} \mathbf{u}(t)
\end{array}\right. \text { The equation of state ca-n be }
$$

expressed by the capacitors, resistors and voltage controlled current source, as Fig.1 shown.

\section{B. Internal Source (IS) sub-model}

IS sub-model is used to describe the active devices, digital, analog and I/O part of the internal source, generally expressed in the form of a current source. IS method for determining the main two ways[7]:

\section{1) Approximate estimate}

In this method, based on semiconductor manufacturing technology, device size, the average peak current of gate level and the average flip door number of approximate estimation device drive current pe-ak. Then estimated the IS based on the operating frequency parameters, Fig 2 shows the IS in the time domain waveform. We can choose to use a peak equal to the saw tooth function and trigonometric functions, analytic form as the outline of the actual current waveform, available PWL piecewise linear current source to approximate the actual current waveform.

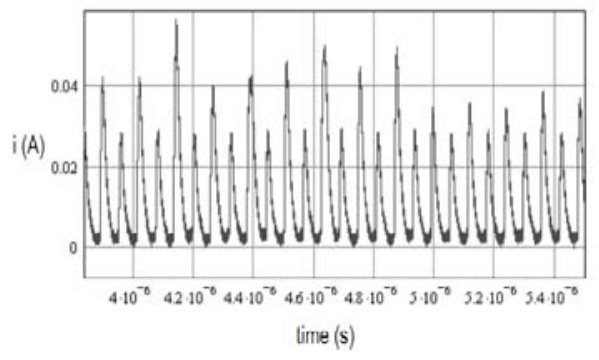

Fig.2(a) The practical time domain waveform of IS
For example, a 16-bit microprocessor in a $32 \mathrm{~nm}$ process, a total of $3000 \mathrm{~K}$ gates, and the operating frequency is $4 \mathrm{MHz}$. Assume each clock edge is ab-out $10 \%$ of the gate of the state flip, from the table 4 we can learned that the average gate current of the chip is about $0.04 \mathrm{~mA}$. Therefore we can calculate the av-ailable chip in the drive current of each clock edge peak is about $3000 \mathrm{~K} * 10 \% * 0.04 \mathrm{~mA}=12 \mathrm{~A}$. Ac-cording to the clock frequency to determine the cur-rent cycle for 246ns (one of a clock cycle gates in the rising and falling edges, have two state flip, each process will have a cur-rent), according to the previous theoretical study there can estimated the 16-bit microcontroller current consumption as same as HC12, copy the initially set to $500 \mathrm{~mA}$ (secondary emission peak is $100 \mathrm{~mA}$ ). Then, in order to accurately describe the emission of these values here adjusted the current to $725 \mathrm{~mA}$ and $200 \mathrm{~mA}$. Likewise, the rise time, peak time and fall time were adjusted to $4 \mathrm{~ns}$, $1 \mathrm{~ns}$ and $4 \mathrm{~ns}$. Piecewise linear current source is an approximation of the simple device current waveform, is also available a more sophisticated method which based on VHDL of current consumption simulation or a complete simulation to improve model-ing accuracy at high frequencies.

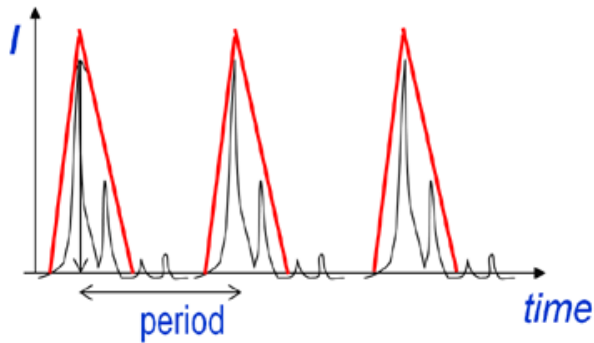

Fig.2(b) According the previous theory estimate the IS waveform in time domain 
TABLE I. IMPACT OF TECHNOLOGICAL SCALE-DOWN ON DIGITAL CIRCUIT CHARACTERISTICS WHICH HAVE AN INFLUENCE ON ELECTROMAGNETIC COMPATIBILITY

\begin{tabular}{|c|c|c|c|c|c|}
\hline Technology & Years & Supply (V) & $\begin{array}{c}\text { Gate } \\
\text { density }(/ \mathrm{mm} 2)\end{array}$ & $\begin{array}{c}\text { Gate current } \\
\text { peak(mA/gate) }\end{array}$ & $\begin{array}{l}\text { Capacity } \\
\text { (fF/gate) }\end{array}$ \\
\hline $1.2 \mu \mathrm{m}$ & 1985 & $5 \mathrm{~V}$ & $8 \mathrm{~K}$ & 1.1 & 60 \\
\hline $0.8 \mu \mathrm{m}$ & 1990 & $5 \mathrm{~V}$ & $15 \mathrm{~K}$ & 0.9 & 40 \\
\hline $0.5 \mu \mathrm{m}$ & 1993 & $5 \mathrm{~V}$ & $28 \mathrm{~K}$ & 0.75 & 30 \\
\hline $0.35 \mu \mathrm{m}$ & 1995 & $5-3.3 \mathrm{~V}$ & $50 \mathrm{~K}$ & 0.6 & 25 \\
\hline $0.25 \mu \mathrm{m}$ & 1997 & $5-2.5 \mathrm{~V}$ & $90 \mathrm{~K}$ & 0.4 & 20 \\
\hline $0.18 \mu \mathrm{m}$ & 1999 & $3.3-2.0 \mathrm{~V}$ & $160 \mathrm{~K}$ & 0.3 & 15 \\
\hline $0.12 \mu \mathrm{m}$ & 2001 & $2.5-1.2 \mathrm{~V}$ & $240 \mathrm{~K}$ & 0.2 & 10 \\
\hline $90 \mathrm{~nm}$ & 2004 & $2.5-1.0 \mathrm{~V}$ & $480 \mathrm{~K}$ & 0.1 & 7 \\
\hline $65 \mathrm{~nm}$ & 2006 & $2.5-0.7 \mathrm{~V}$ & $900 \mathrm{~K}$ & 0.07 & 5 \\
\hline $45 \mathrm{~nm}$ & 2008 & $1.8-0.8 \mathrm{~V}$ & $2000 \mathrm{~K}$ & 0.05 & 3 \\
\hline $32 \mathrm{~nm}$ & 2010 to now & $1.8-0.8 \mathrm{~V}$ & $3000 \mathrm{~K}$ & 0.04 & 2 \\
\hline
\end{tabular}

2) measurement methods

IS description of the internal current of the dev-ice and therefore it can only be obtained by indirect measurement method, the measurement method are as follows:

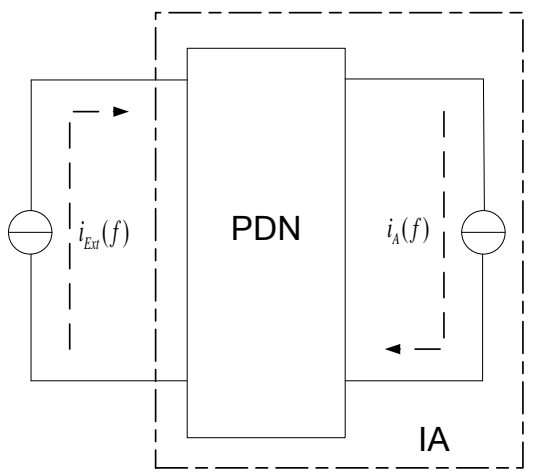

Fig.3 The relationship $i_{E x t}(f)$ and $i_{A}(f)$ the $i_{A}(f)$ can be calculated by measurement the $i_{\text {Ext }}(f)$

If the PDN net is known, then the IS can be obtained by measuring the $i_{E x t}(f)$.The relations between $i_{E x t}(f)$ and $i_{A}(f)$ is:

$$
i_{A}(f)=[P D N] \cdot i_{E x t}(f)
$$

For multiport:

$$
\left[\begin{array}{c}
i_{A[n]}(f) \\
\cdots \\
i_{A[1]}(f)
\end{array}\right]=[P D N] \cdot\left[\begin{array}{c}
i_{E x t[n]}(f) \\
\cdots \\
i_{E x t[1]}(f)
\end{array}\right]
$$

\section{MODELING EXAMPLE}

In order to verify the modeling theory, there use an integrated amplifier to modeling, the package as shown below:

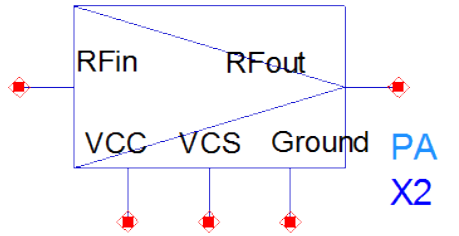

Fig.4 The diagram of the amplifier package The internal circuit as shown below:

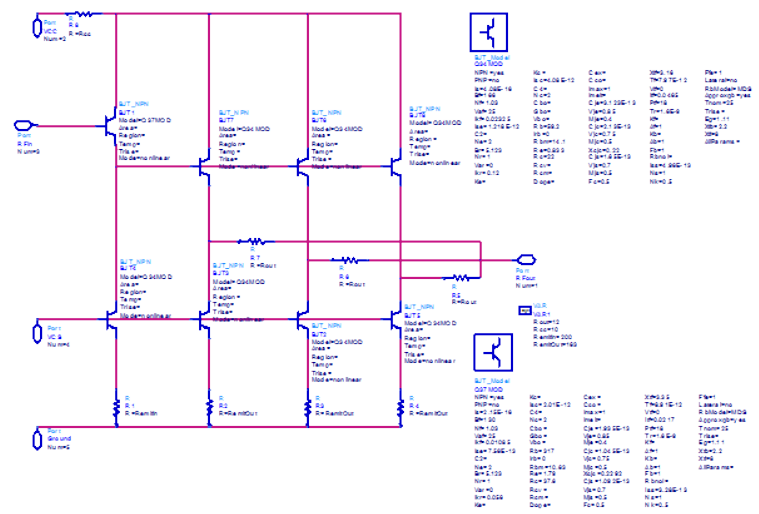

Fig.5 The internal circuit of the amplifier

Assume we didn't know the internal circuit of this device then uses the theory that put forward and simulation the device's Y-parameters of the supply network, the schematic circuit is as Fig.6 shows: 


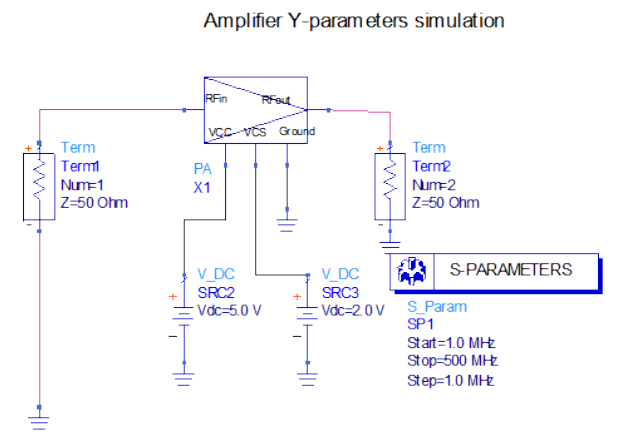

Fig.6 Using ADS simulator set up a measurement circuit to get the I/O Y-parameter

Fig.7 is the simulation results,(a) is the simulation result in ADS simulator and (b) is the fitting result, among this figure the green line is the relative error, as this figure shows we can get that the fitting result is accurate.

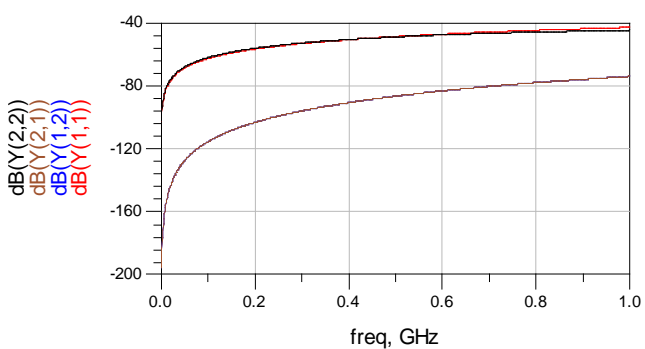

(a)

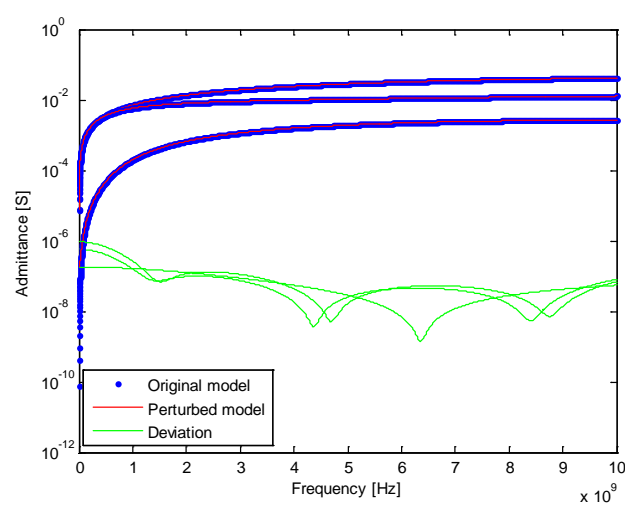

(b)

Fig.7 The simulation results, (a) is the simulation result in ADS simulator and (b) is the fitting result use VF technology

Then use the vector fitting technique fitting the data and built the two-port SPCICE circuit netlist:

*Ypara2

*Lumpled Circuit Generated indirectly from spacevariable equations by VF

.subckt Ypara2 12

Rd1 $100 \quad 10.514701$

G1_2 $10 \begin{array}{llll}0 & 0 & -0.012996929\end{array}$

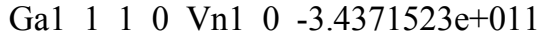

$\mathrm{Ga} 2-1 \quad 1 \quad 0 \quad \mathrm{Vn} 2 \quad 0 \quad-38554522$

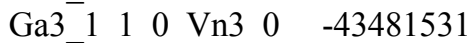

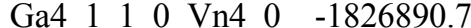

Ga5_1 1100 Vn5 00.016624339

Ga6 1110 Vn6 $0 \quad 4.81222 \mathrm{e}+010$

Ga7_1 10 Vn7 $0 \begin{array}{rlll}0 & 0 & -1000102 \mathrm{e}+008\end{array}$

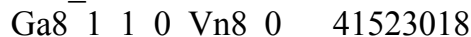

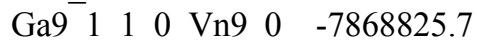

Ga10_1 10 Vn10 $0 \quad 0.21522103$

$\mathrm{Rd} 2 \overline{2} 0 \quad 4.9696399$

G2 $1 \begin{array}{llllll}1 & 2 & 0 & 1 & 0 & -0.012996929\end{array}$

$\mathrm{Ga} 1 \quad 220 \quad \mathrm{Vn} 1 \quad 0 \quad 4.81222 \mathrm{e}+010$

Ga2_2 20 Vn2 0 - $1.1000102 \mathrm{e}+008$

To check the veracity of the fitting result. Reputing the netlist into ADS to simulation the circuit is shown below:

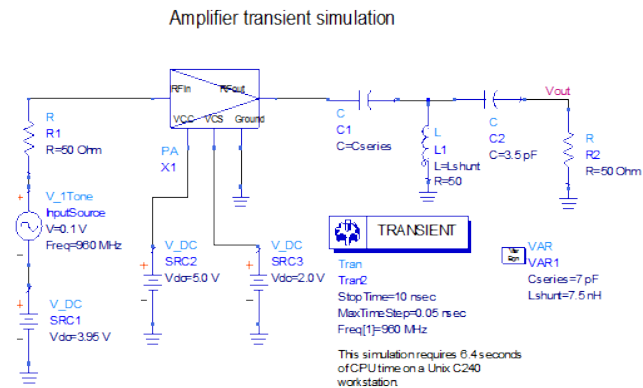

(a)

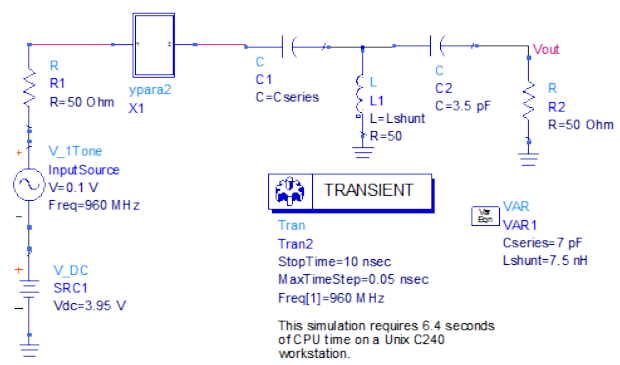

(b)

Fig. 8 The simulation circuit, (a) is the simulation in ADS simulator using the original device and (b) is the simulation in ADS simulator using the behavior model that builds

The simulation contrast result between the amplifier and the model that we build is shown in Fig.9. By comprehensive analysis, that the theory which we proposed before can rebuild the active device accurately.

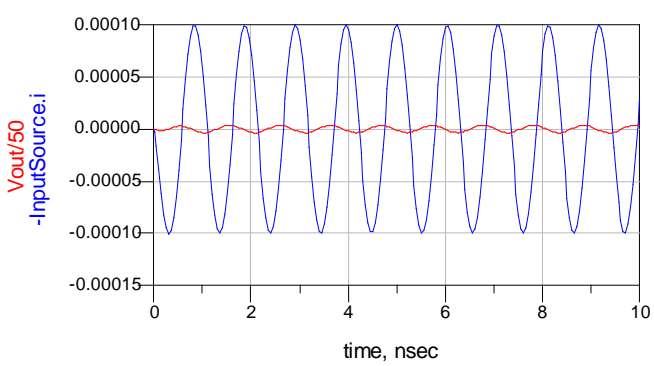

(a) 


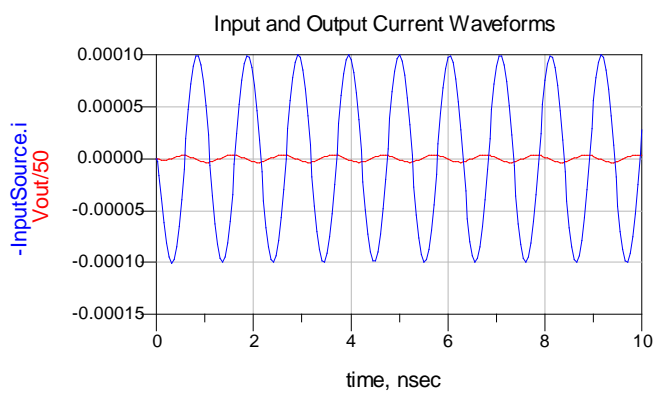

(b)

Fig.9 The simulation result, (a) is the result using the original device and (b) is the result using the behavior model that builds

\section{CONCLUSION}

This paper has report a theory of modeling active device broadband conducted emission behavior model based on vector fitting theory. The passive distribution network is characterized by means of Y-parameter measurement. The internal source is modeled by a current source whose waveform is modeled by approximate estimate or measurement methods

\section{REFERENCES}

[1] Cai Xing-jian and Mao Jun-fa, "Transient BehavioraI Modeling of High-Speed DigitaI I/O Buffer Based on IBIS Model" Journal of shanghai jiaotong university. china, vol. 35 No.1, pp. 5-9, Jan. 2001.

[2] Gustavsen B., Semlyen A. Rational approximation of frequency domain responses by vector fitting[J]. IEEE Transactions on Power Delivery, 1999,14(3):1052-1061

[3] Swaminathan M., Engin A. E. Power Integrity Modeling and Design for Semiconductors and Systems[M]. $2009: 56-70$, 163-195

[4] Semlyen A., Gustavsen B. A Half-Size Singularity Test Matrix for Fast and Reliable Passivity Assessment of Rational Models[J]. IEEE TRANSACTIONS ON POWER DELIVERY, 2009, 24(4):345-351

[5] Gustavsen B. Fast Passivity Enforcement for Pole-Residue Models by Perturbation of Residue Matrix Eigenvalues[J]. IEEE TRANSACTIONS ON POWER DELIVERY, 2008, 23(4) :2278-2285

[6] Gao S., Li Y. S., Zhang M. S. An Efficient Algebraic Method for the Passivity Enforcement of Macromodels[J]. IEEE TRANSACTIONS ON MICROWAVE THEORY AND TECHNIQUES, 2010, 58(7):1830-1839

[7] International Electro-technical Commission, IEC 62433-2 "Models of integrated circuits for EMI behavioural simulation Conducted emissions modelling (ICEM-CE)”, 2008 